\title{
Mobile Application Based Heavy Vehicle Fatigue Compliance in Australian Operations
}

\author{
Luke Mirowski and Joel Scanlan \\ School of Technology, Engineering and Design, University of Tasmania (UTAS) \\ Hobart, Australia \\ \{Luke.Mirowski, Joel.Scanlan\}@utas.edu.au
}

\begin{abstract}
The Australian National Heavy Vehicle Regulator (NHVR) defines the rules for fatigue management in heavy haulage trucking operations. The rules place restrictions on total work and minimum rest hours, and are aimed at regulating the potential for fatigue risk amongst drivers. This paper presents a performance-based fatigue management system based on driver fatigue data stored in simple mobile databases and deployed via Android smart phones. The system funded by WorkSafe Tasmania and entitled, Logistics Fatigue Manager (LFM), was evaluated with a cohort of heavy haulage drivers in Australian forestry. The correlation between driver fatigue estimates and actual sleep hours (recorded using FitBits) is confirmed, and is also supported through driver interviews. The benefit is that management of fatigue risk could be more tailored to individual drivers opening up efficiency gains across supply chains.
\end{abstract}

Keywords: Data Entry, Mobile Databases, Data Heuristics

\section{Introduction}

This research project funded by WorkSafe Tasmania aligns with compliance requirements of the Australian Heavy Vehicle Driver Fatigue laws from the National Heavy Vehicle Regulator (NHVR) [1], which stipulates all stakeholders in a supply chain which uses heavy haulage trucks must comply with standard, basic and advanced fatigue management rules.

There are two elements to the legislation: regulated work and rest hours, and work diary reporting; and secondly, a general duty element. Firstly, the laws regulate work and rest hours for heavy vehicle drivers. All parties in the supply chain are legally responsible for preventing drivers from becoming fatigued (i.e. tired through lack of rest). Secondly, all reasonable steps must be taken to enable drivers to manage and recover from fatigue, which means: drivers must stop if fatigued; operators and schedulers planning a driver's rest breaks; loading managers ensuring queuing is managed to allow for efficient truck movement; and contracts requiring drivers to break the law are illegal. The current approaches are paper based with data residing in paper based and offline storage.

The aim of this research project is to trial and evaluate a performance-based ap- 
proach to heavy haulage driver fatigue management that evaluates individual driver fatigue, and improves safety standards by integrating heavy vehicle fatigue management regulations, transport scheduling requirements and driver rostering principles in an electronic system based on simple mobile databases, apps and fatigue risk models.

The system developed in the project has been primarily developed for and deployed within Tasmania's forestry industry heavy haulage operations to test the feasibility of the technology and the accuracy. The objective has been to first build a technologically innovative fatigue risk management system that is capable of assisting drivers to comply and exceed safety standards within the Australian forestry industry and transportation sector. The software, Logistics Fatigue Manager (LFM), and all the tools it comprises of, are available online at www.logisticsfatiguemanager.com.au.

\section{Background}

Heavy vehicles are the predominant transport mode in use in Australia for land based freight tasks [2]. Sustainability of these transport operations, from a supply chain management and logistics perspective, relies on finding appropriate levels and combinations of many operational factors, all of which impact on safety and cost. The optimal combination of factors is difficult to achieve as factors are selected to minimise cost in preference to fatigue risk management which often requires stoppages and breaks in driving.

Australian fatigue laws now include all the personnel and all the parties involved in the chain of responsibility to be made liable for fatigue if they influence the driver's activities. This challenging when data resides in paper based and offline storage.

'Standard Hours' guidelines focuses on having a minimum rest (driver must take a break for at least 30 minutes in 5 hours interval during their work, for example) and having a maximum upper-limit on work hours, they also provide basic record keeping requirements which are suitable for the most of the businesses but which are paper based [3][4].

Under the current paper based system, for productivity of heavy vehicle truck drivers, one of the major challenges is the truck driver's hours of work are reduced which will lead to significantly less payment for a truck driver. Whereas the truck driver may be capable of driving 14 hours, the regulations mean the truck driver, under a standard hour's operation, can only drive 12 hours in a day. This means the truck driver is working 2 hours less per day, 14 hours less per week. [5]

To resolve these challenges, this research applies a performance based approach to fatigue assessment by way of an electronic system supported by a mobile apps, databases and applications. It leverages data captured through a mobile app and data stored on the phone to calculate a driver's individual fatigue risk score.

\section{Method}

The research consists of two parts: development of the performance-based fatigue system, and a trial/evaluation period spanning two phases. 


\subsection{Software Development}

The software tool, an android mobile application (the 'LFM for the Driver' mobile application), was installed on driver electronic tablet devices. The software eliminates identified deficiencies in the physical work-diary formats being used currently by drivers by providing a more efficient method to capture, collect and distribute data:

- Driver reading/writing deficiencies: the software enables drivers to record work and rest periods on their tablets using a simple button interface.

- Cost of specialised equipment: the software is capable of operating on any standard Android mobile phone or tablet, utilising its inbuilt GPS, Wi-Fi.

- Fatigue assessment: the software runs a fatigue score calculator in the background, which keeps a history of the driver's fatigue level based on data captured.

The simple mobile database underpinning the system enables low-cost localised monitoring, management and support for drivers whilst an online dashboard and database allows data to be shared with supervisors and managers. Options in the software allow for mediation of access to database data across the organisation and supply chain.

\subsection{Data Collection}

Heavy haulage truck drivers from Australian forestry volunteered from several companies to be part of the trial and evaluation of the LFM system.

\section{Phase One}

The drivers used an Android tablet to record estimates of their sleep periods in 24 and 48-hour periods before commencing a working shift with data stored locally and remotely. They also recorded the periods and time of rest along with then vehicle's odometer readings in the 'LFM for the Driver' app.

\section{Phase Two}

The methodology conducted for collating data remained largely the same. However, it was supplemented by the use of the FitBit device as secondary option of verifying driver sleep information.

\section{$4 \quad$ Results and Discussion}

Both quantitative and qualitative data was collected from the drivers involved in the trial and evaluation of the system across the phases. These are now reported.

\subsection{Quantitative Results}

\section{Estimation of previous sleep before work}

The graph in Figure 1 represents information from the LFM app that correlates the drivers' estimated sleep hours and the start of shift fatigue score, confirming drivers are good at estimating their prior sleep hours through the app. 


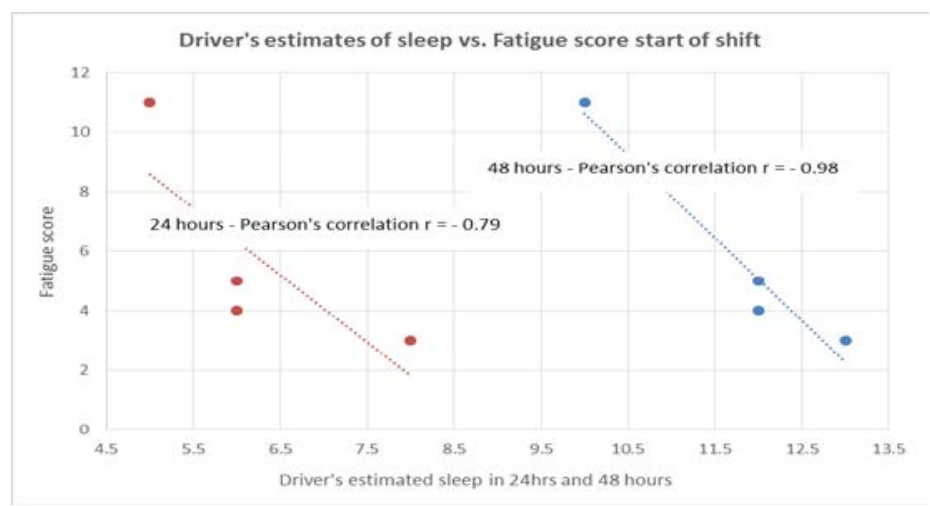

Fig. 1. Driver’s 24-hour \& 48-hour sleep estimation vs. Fatigue scores before work.

The findings confirmed through correlation that the driver's sleep estimates and the sleep hours recorded on the FitBit device were similar. The data confirmed that the individual drivers estimated their time spent asleep with a fair amount of accuracy, proving the reliability of a fatigue score rating collected through the app.

\section{Managing fatigue during work periods}

Figure 2 confirms the relationship between the drivers' work and rest activity and dynamic fatigue score calculated throughout day 1 (as an example). Dynamic fatigue levels increase when mandated rest periods are not taken during a working shift. Driving for five and a half hours continuously without a rest period increases the dynamic fatigue score. The driver then worked for an additional $4 \frac{1}{2}$ hours without rest. The graph registers a significant increase in the dynamic fatigue score. This confirms the electronic system can model work and rest from the drivers' data, with a fatigue risk model score, reflecting driver accumulation and recovery from fatigue.

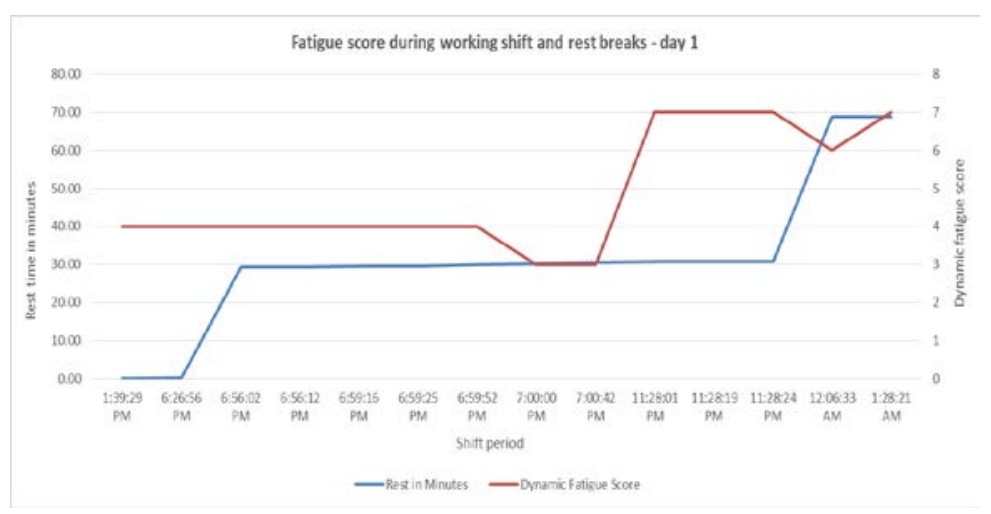

Fig. 2. Fatigue \& Rest Breaks - Shift 1. 


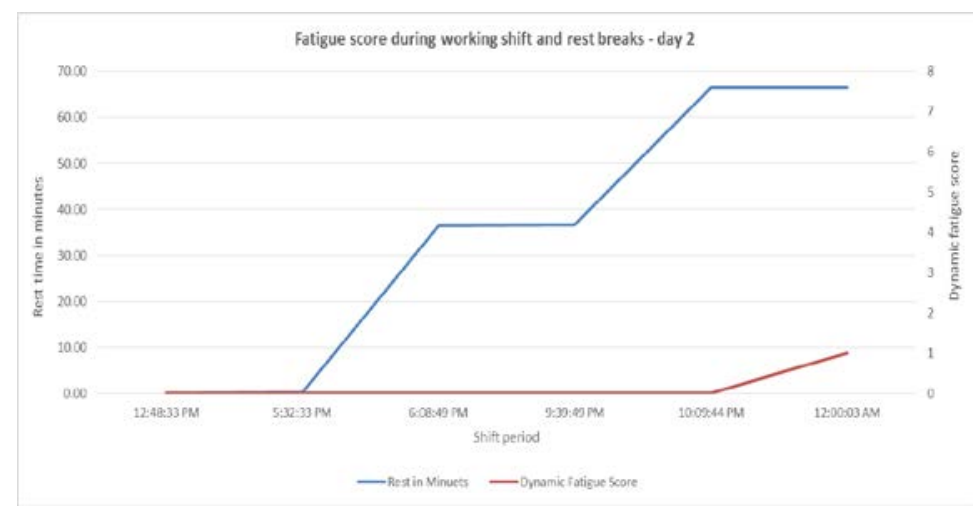

Fig. 3. Fatigue \& Rest Breaks - Shift 2.

Figure 3 demonstrates the relationship between the drivers' work and rest activity and dynamic fatigue score throughout the working of day 2. The dynamic fatigue score at the start of the working shift was low. At the end of the working shift the fatigue score began to increase because (a) the shift was 12 hours and hence longer, and (b) the rest taken at the end of a shift did not compensate for the extended hours. Again, this confirms that the electronic system can model fatigue risk interrelationships.

The data for day three (not shown) was similar to day two, at the start of the working shift the dynamic fatigue score is low. This low fatigue score is maintained throughout the day by (a) taking the mandated rest periods and, (b) taking the rest in the stipulated intervals. The data for the fourth day (not shown) shows that the driver is set into a routine and which continues to confirm the above relationships.

For the trial and evaluation periods, most of the data from across all the days again confirms correlation of fatigue scores with work and rest hours in the electronic system. Hence a simple mobile app and collected data combined with a fatigue risk model is an effective approach to capturing performance based fatigue risk information.

\subsection{Qualitative Results}

Trial participants were also interviewed during the project to establish qualitative factors to successfully managing fatigue risk through the LFM system and these are now reported.

\section{Self-management of fatigue}

Most drivers considered themselves to be the best judge of when they felt fatigued during the work shift. This misconception of self-regulating their rest breaks arose from their personal interest and that of the principal contractors. Though drivers complied with mandated rest breaks to a fair extent, they did take the liberty of selfmanaging fatigue during a shift. The system was confirmed as helpful to this process by the drivers as distinct from a paper diary through localized fatigue risk management via their smart phones (and the data stored locally to them). 


\section{Electronic system for fatigue management}

Most drivers were not averse to have some form of monitoring and reporting for fatigue management, as it created self-awareness of the rest required and also demonstrated to employers the need for rest breaks during extended periods of driving. Other variables, such as time of day or night, route and payload, were highlighted as factors that impacted fatigue levels and essential to achieving accurate estimates of fatigue levels in any assessment period. As these factors were collected using the LFM system, drivers perceived the LFM system to be robust at collecting fatigue data.

\section{Conclusions and Further Work}

In this paper the main outcomes of the trial and evaluation of an electronic fatigue management system, entitled Logistics Fatigue Manager (LFM), were reported. This project was funded by WorkSafe Tasmania.

During the project, quantitative data was collated on heavy vehicle driver fatigue in a forestry supply chain, using the Logistics Fatigue Manager (LFM) system. Correlated results from the LFM system and FitBits confirmed that drivers could make reliable estimates of their sleep hours from which fatigue risk scores could be calculated.

Qualitative interviews were conducted to contextualize and support the findings. There were unanimously favorable response to the electronic system and active participation in feedback for future improvements to the electronic system.

The results suggest that simple mobile-deployed databases and apps combined with fatigue risk models could generate effective data collection opportunities to shift fatigue risk management in Australian heavy haulage operations from compliancebased to performance based modes of operation.

This in turn could increase accuracy and speed of reporting, as well as create opportunities to focus on managing individual 'customised' driver risk profiles.

Further work may consider options for the widespread collection and collation of heavy haulage driver data through smart phones and mobile databases for low-cost and performance-based management of heavy haulage driver fatigue risks.

\section{References}

1. NHVR. Basic Fatigue Management. 2016; Available from: https://www.nhvr.gov.au/safety-accreditation-compliance/fatigue-management/workand-rest-requirements/standard-hours.

2. Department of Infrastructure and Regional Development-Australian Government, Freightline 1-Australia freight transport overview. 2014.

3. NHVR. Electronic Work Diary. 2016; Available from: https://www.nhvr.gov.au/safetyaccreditation-compliance/fatigue-management/electronic-work-diaries-ewds.

4. NHVR. Chain of Responsibility. 2015; Available from: https://www.nhvr.gov.au/safety-accreditation-compliance/chain-of-responsibility.

5. Goel, A., et al., Truck driver scheduling in Australia. Computers \& Operations Resarch, 2012(39): p. 1122-1132. 